\title{
A Business Application of the System Dynamics Approach: Word-of-Mouth and Its Effect in an Online Environment
}

\author{
Roman Wong and Shirley Ye Sheng
}

\author{
"( To profit from good advice requires more wisdom") \\ than to give it. \\ John Churton Collins (1848-1908) \\ Author and literary critic
}

\begin{abstract}
In this article, we illustrate the use of system dynamics modeling approach to study a complex system: word-of-mouth. Word-of-mouth plays an important role in reducing risk and uncertainty in purchase and consumption. Most of the prior research on word-of-mouth focused on studying either the factors that trigger consumers' participation (sending or receiving) in word-of-mouth activities or the impact word-of-mouth information has on consumers' buying decisions. The relationship between the two decision processes, however, is recursive and dynamic. Most prior studies have not focused on a recursive relationship. Our objective is to present a system dynamics model for the study of the relationship between the buying decision and the decision to participate in word-of-mouth communication. We also discuss how system dynamics modeling can be used in other complex problems in business such as the creation of a global business.
\end{abstract}

\section{Introduction}

Word-of-mouth plays a crucial role in helping to reduce risk and uncertainty in purchase and consumption (Murray and Schlacter, 1990; tinyurl.com/7cylp66). It is an informal, customer-to-customer communication about the characteristics of a business or a product. The objective of the study described in this article is to construct a computational architecture and use it as a simulation tool for the study of the dynamic recursive relationship between consumers' decision to adopt a product and how the adoption experience may trigger their contribution of word-of-mouth messages to online review websites, which frequently exert impacts on other potential adopters.

Prior research on word-of-mouth has mainly focused on studying either the factors that trigger consumers' participation (sending or receiving) in word-of-mouth activities (e.g., Dellarocas et al., 2004: tinyurl.com/7rhysge; Godes and Mayzlrrin, 2004: tinyurl.com/7fu6nja) or the im- pact word-of-mouth information has on consumers' buying decisions (e.g., Chatterjee, 2001: tinyurl.com/ 7avb5th; Chen and Xie, 2005: tinyurl.com/6rs5cu2; Chevalier and Mayzlin, 2006: tinyurl.com/7bqjysz). However, the buying decision and the decision to participate in word-ofmouth communication are not disjoined and not much has been done to study the interdependency existing in this relationship. Our study contributes to the existing literature by providing insights into the interactive dynamics of the two processes: the process of how wordof-mouth affects online shoppers' decision making for product adoption.

In this article, we briefly discuss the nature of the two decision processes involved in word-of-mouth and their inter-relationship. Next, we present our systemdynamics model for adoption and word-of-mouth. We then draw out the implications of this research for global business creation. Finally, we discuss some future directions to further our current research. 


\section{Word-of-Mouth and Its Effect in an Online Environment}

Roman Wong and Shirley Ye Sheng

\section{Word-of-Mouth}

Word-of-mouth can take one of many forms. An online review from another customer on a shopping website is a common example. Consumers also often acquire information about specific products from various online communities such as blogs and product review websites. Studies have shown that consumer reliance on word-of-mouth is increasing. For example, the ongoing study conducted by the Kokokusha group (2007; tinyurl .com/7ulr6f4) has shown that such reliance increased from $61 \%$ in 2005 to $76 \%$ in 2007 ).

Prior studies (e.g., Chen and Xie, 2005: tinyurl.com/6rs5cu2; Bone, 1995: tinyurl.com/7ttvzfd) show evidence that suggests consumers tend to rely on other people's experiences and opinions during the decision-making process when purchasing a high-involvement product or service. This is especially the case when: i) the transparency of the product is high; ii) the product is complicated; iii) the criteria for an objective evaluation of the product are difficult to assess; and iv) the perceived risk is high.

In some cases, e-shoppers who have made an adoption decision may wish to contribute an online review about the adoption experience regarding the product they adopted. Such contributing activities allow consumers to exert both informational and normative influences on the product evaluation and purchase intention of fellow consumers (Bone, 1995: tinyurl.com/7ttvzfd; Ward and Reingen, 1990: tinyurl.com/6nvsr2p). Past research has linked word-of-mouth activities to factors including satisfaction, loyalty, quality, commitment, trust, and perceived value.

Harrison-Walker (2001; tinyurl.com/7movns7) suggests that word-of-mouth valence (i.e., whether a review is positive or negative) is an important dimension that may exert a significant impact on buying decisions. Figure 1 indicates major contributing factors that lead to consumer participation in word-of-mouth activities. Figure 2 is a model showing word-of-mouth as a significant factor that influences a shopper's buying decision and moderates the impacts of other factors.

An online shopper's product-adoption decision making, however, has an interrelated connection with their decisions about making contributions to word-ofmouth. There is a recursive relationship between the two decision processes in that the buying decision of a consumer, which leads to the later evaluation of the purchased product, may then lead to the decision of

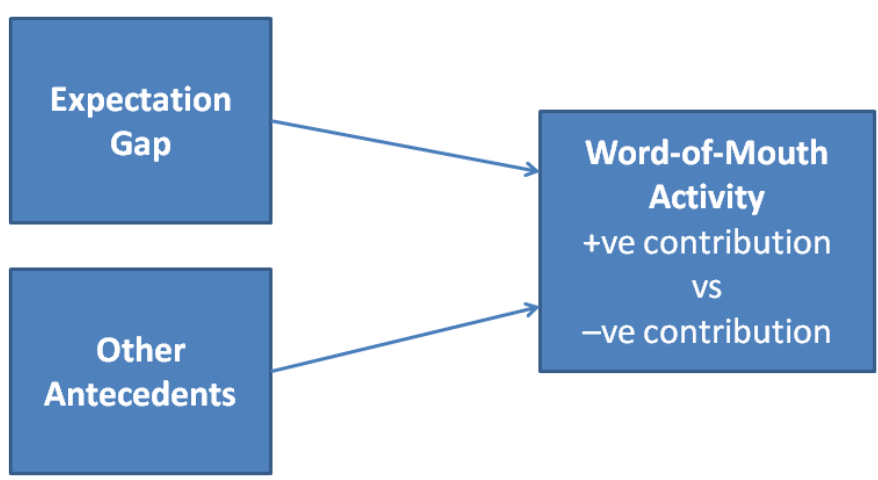

Figure 1. Contributing factors of word-of-mouth activity

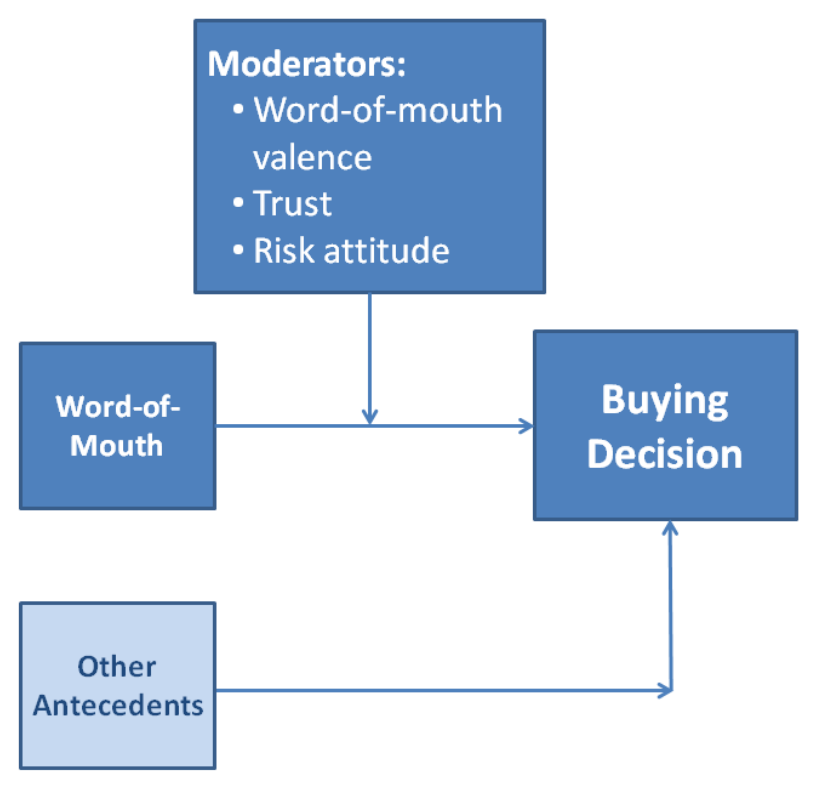

Figure 2. A model for word-of-mouth impact on a buying decision

whether or not to send out word-of-mouth in regard to the consumer's experience with the product. The consumer's word-of-mouth contributions in turn may likely influence the buying decisions of other consumers in the online community. Figure 3 indicates such recursive and dynamic influence between the buying decisions and the decisions to send a word-ofmouth message. So far, there has not been much research devoted to such recursive relationship and the dynamic nature of these two decision processes. In the current study, we concentrate on studying the recursive relationship between the buying decision and the decision to participate in word-of-mouth communication. 


\section{Word-of-Mouth and Its Effect in an Online Environment}

Roman Wong and Shirley Ye Sheng

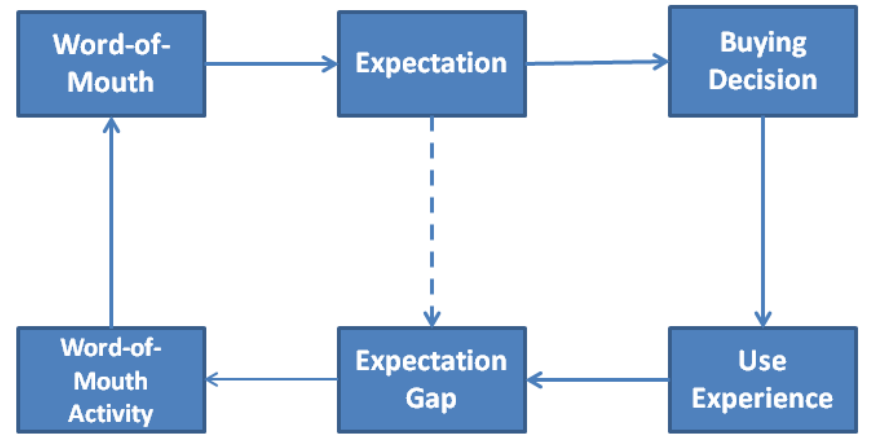

Figure 3. The recursive relationship between consumers' buying decisions and their word-of-mouth activity decisions

\section{A System-Dynamics Model for Adoption and Word-of-Mouth}

System dynamics is "an approach to understanding the behaviour of complex systems over time" (tinyurl.com/ yrqbyx). In this section, we present a system-dynamics model that we constructed to explain the recursive relationship between online shoppers' buying decisions and their activities of sending word-of-mouth messages.

\section{Causal loop between market saturation and word-of-} mouth

In system dynamics modeling work, causal-loop diagrams (CLDs) are frequently used to represent the stocks and flows in a system. CLDs indicate how interrelated variables impact one another (Richardson and Pugh, 1981: tinyurl.com/ccco9zn; Kim, 1992: tinyurl.com/ bwsxl4e).

The CLD in Figure 4 provides a representation of the two phenomena we included in our word-of-mouth and adoption model. The left-hand-side loop of the CLD represents how the change in the number of potential adopters impacts the adoption rate, which in return causing a change to the balance of the potential adopters. The causal loop is balancing by nature and labeled as 'Market Saturation'. The right-hand-side loop in Figure 4, on the other hand, represents how the change in the adoption rate brings along a change in the number of adopters, which in turn exerts an exponential impact, either positive or negative, depending on the direction of the original change in the adoption rate, on the adoption rate itself. Such a causal loop is reinforcing by nature and labeled as "Word of Mouth".

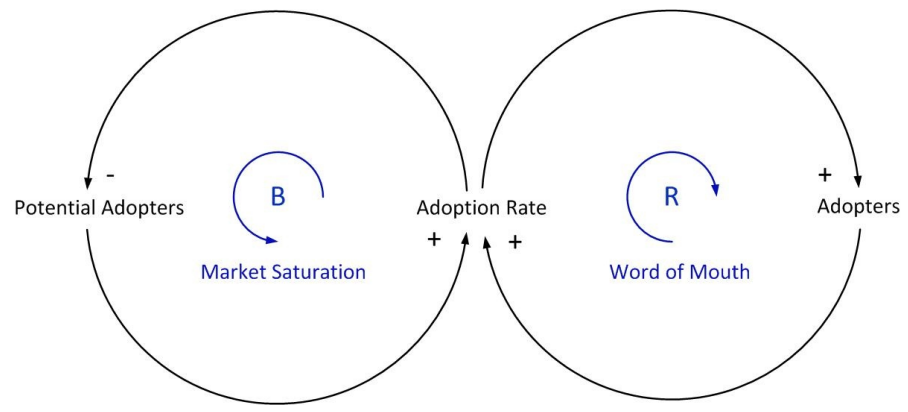

Figure 4. A causal-loop diagram indicating the dynamic relationship between adoption and word-of-mouth

\section{Implementation}

Our word-of-mouth system-dynamics model was implemented using NetLogo (ccl.northwestern.edu/netlogo/), a freeware software package developed by Uri Wilensky of Northwestern University. NetLogo has been more frequently used for modeling agent-based problems, however the software comes with a programming tool called 'Systems Dynamic Modeler', which was designed for system dynamics modeling problems. We used System Dynamics Modeler for our aggregate system model, and we used NetLogo for our agent-level modeling in the second phase of our study.

Figure 5 depicts the key stocks (rectangular shapes), flows (broad arrow with faucet shape), and links (arrowed arc line) in the system-dynamics model for the word-of-mouth and adoption decision processes.

A stock is a collection of things, an aggregate, or a state variable. For example, a stock can represent a population of online shoppers, a collection of word-of-mouth messages, or the number of adopters. A flow brings things into, or out of, a stock. Flows look like pipes with a faucet because the faucet controls how much stuff passes through the pipe. Examples include decision to buy made by the online shoppers. A link makes a parameter value from one part of the diagram available to another. A link transmits a number from a variable or a stock into a stock or a flow.

There are four stocks identified for our model: Adopter (adopter), Potential Adopter (shopper), Positive Wordof-Mouth (PWOM), and Negative Word-of-Mouth (NWOM). Both Adopter and Potential Adopter have 


\section{Word-of-Mouth and Its Effect in an Online Environment}

\section{Roman Wong and Shirley Ye Sheng}

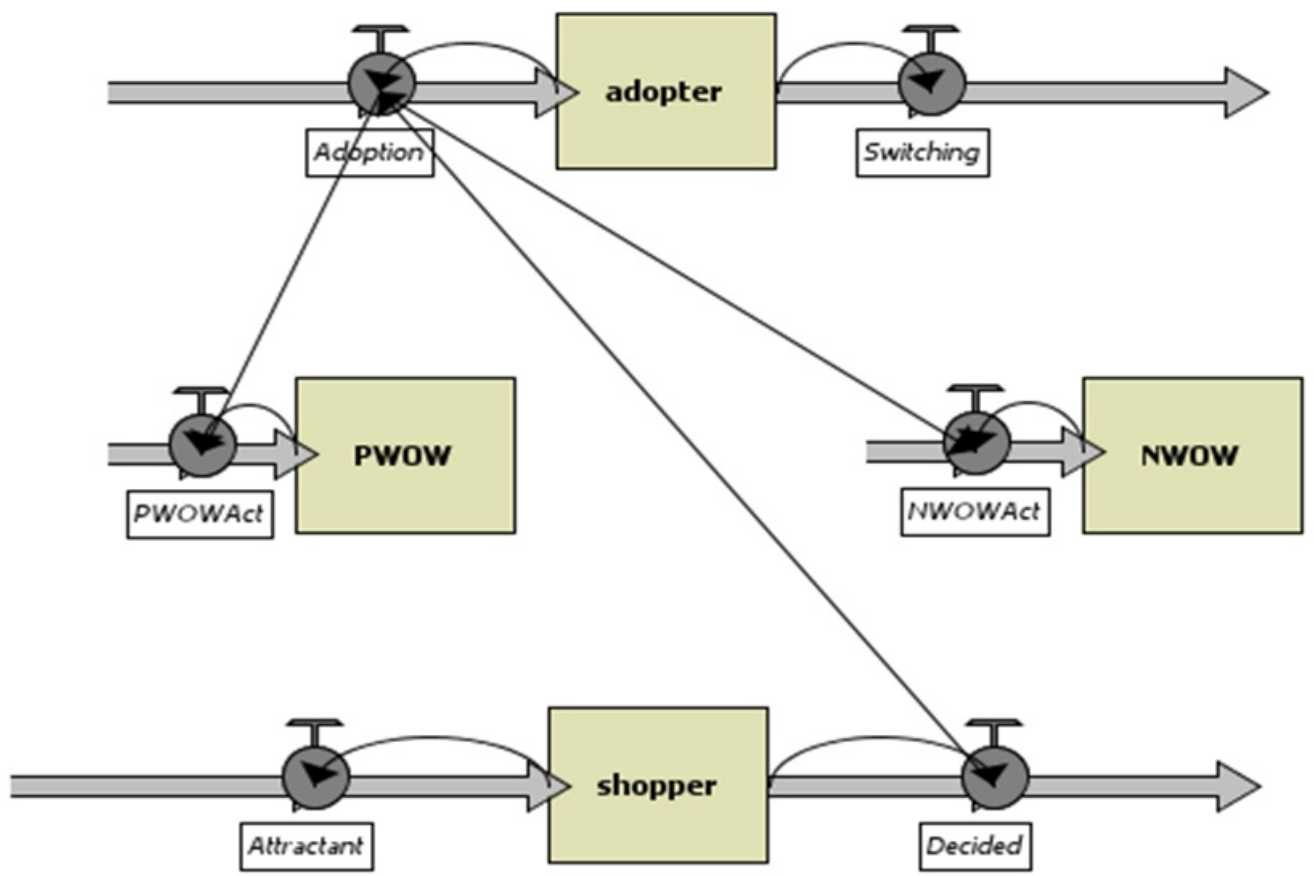

Figure 5. Major system components implemented in the system-dynamics model

flows into them and flows out of them. For the Positive WOM and Negative WOM stocks, there are only inward flows.

For Adopter, the inward flow is Adoption (i.e., when a Potential Adopter decides to buy). The flow pattern for this inward flow follows a gamma distribution as is commonly observed in new product adoption cases (see Figure 6). The mathematical representation for the in-flow of Adoption is:

$$
\text { Adoption }=\text { shopper }{ }^{k(p w o m ~} /\left(\text { pwom }+a^{*} \text { nwom }\right)
$$

where $\mathrm{k}$ is a scalar constant that defines the shape of the distribution, and where $\alpha$ is a multiplier constant representing how sensitive the Potential Adopter is to the Negative WOM. There is an outward flow "Switching" that causes Adopter to decrease. Switching represents the depletion in number of Adopter due to switching to other products or end of product life cycle. Its outflow rate is a function of the number of Adopter and a switching rate.

For Potential Adopter, the in-flow is the amount of potential adopters newly attracted (Attractant) to consider adopting the product. The flow rate is represented as a function of Adoption and a scalar constant (attract-
Rate). The outward flow of Potential Adopter is the amount of potential adopters that decided to adopt the new product (i.e., Adoption). The mathematical representation for the stock of potential adopters (shopper) is as follows:

$$
\text { Shopper }=a+\text { Attractant }- \text { Adoption }
$$

where $a$ is the initial number of potential adopters to start with.

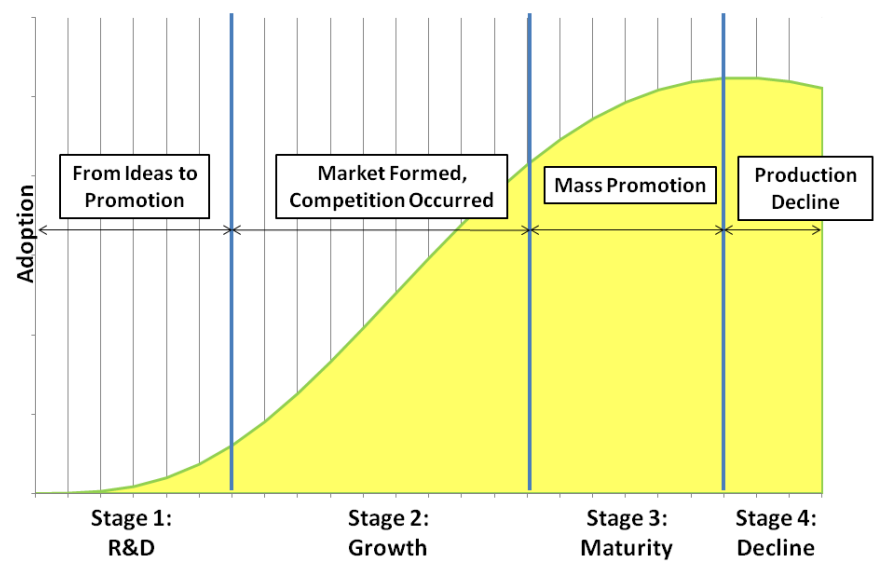

Figure 6. New product and technology adoption lifecycle 


\section{Word-of-Mouth and Its Effect in an Online Environment}

Roman Wong and Shirley Ye Sheng

For the Positive WOM (PWOM), the in-flow is the amount of number of new positive word-of-mouth messages contributed by adopters. The flow rate is represented as a function of Adoption and a scalar constant (pwomRate). The mathematical representation for PWOM is as follows:

$$
P W O M=b+\text { Adoption * pwomRate }
$$

where $b$ is the initial number of PWOM to start with.

For the Negative WOM, the in-flow is the amount of number of new negative word-of-mouth messages contributed by adopters. The flow rate is represented as a function of Adoption and a scalar constant (nwomRate). The mathematical representation for NWOM is as follows:

$$
\text { NWOM }=c+\text { Adoption * nwomRate }
$$

where $c$ is the initial number of NWOM to start with.

In addition to the System Dynamics Modeler, NetLogo also provides a set of tools for creating fundamental user interface for input and controls. For the current exercise, the slider control allows the model users to enter parameter values in variable rates. The textbox control can be used for input or output purposes. The button control provides the model users means to activate a procedure. Figure 7 shows a variety of controls for user interface of the model.

\section{Result for sample configuration}

The product adoption and word-of-mouth system-dynamics model in this study is a system of coupled, nonlinear, first-order differential equations. The simulation is implemented by dividing a time unit into discrete in- tervals of length (dt) and stepping the system through time one $\mathrm{dt}$ at a time such as the following generic format:

$$
\frac{d}{d t} \mathbf{x}(t)=\mathbf{f}(\mathbf{x}, \mathbf{p})
$$

where $\mathrm{x}$ is a vector of levels (stocks or state variables), $\mathrm{p}$ is a set of parameters, and $\mathrm{f}$ is a nonlinear vector-valued function. In our model, the differentiation process starts with the stock adopter, followed by potential adopter, and then the two kinds of word-of-mouth. Applying the differentiation to our current model, $f$ is the function that determines the amount of adoption, while $\mathrm{x}$ is the number of shoppers (i.e. potential adopters) and the parameter $\mathrm{p}$ being the compound exponent index (k(pwom / (pwom $+\alpha^{*}$ nwom) ) in equation (1).

In most new product adoption cases, the distribution of such new product adoption throughout its life-cycle usually follows a gamma pattern. As such, the mathematical characteristics of our model demonstrate a similar pattern of that of a gamma distribution pattern. Based on our initial observation of the online adoption behaviors for a certain model of digital camera on a leading shopping website, we derived a sample of parameter values to configure our adoption and word-ofmouth system dynamics model.

Theoretically, the scalar constant $k$ and the multiplier constant $\alpha$ are market specific. For our initial model, we proxied the number of shoppers $(x)$ by the number of browsers visiting the online review board. We use 0.8 and 0.2 for $k$ and $\alpha$ respectively as the default values in lieu of values more specific for the digital camera. The default values have been tested on a number of products and found to be reasonably representative. The two variables, pwom and nwom, are dynamically

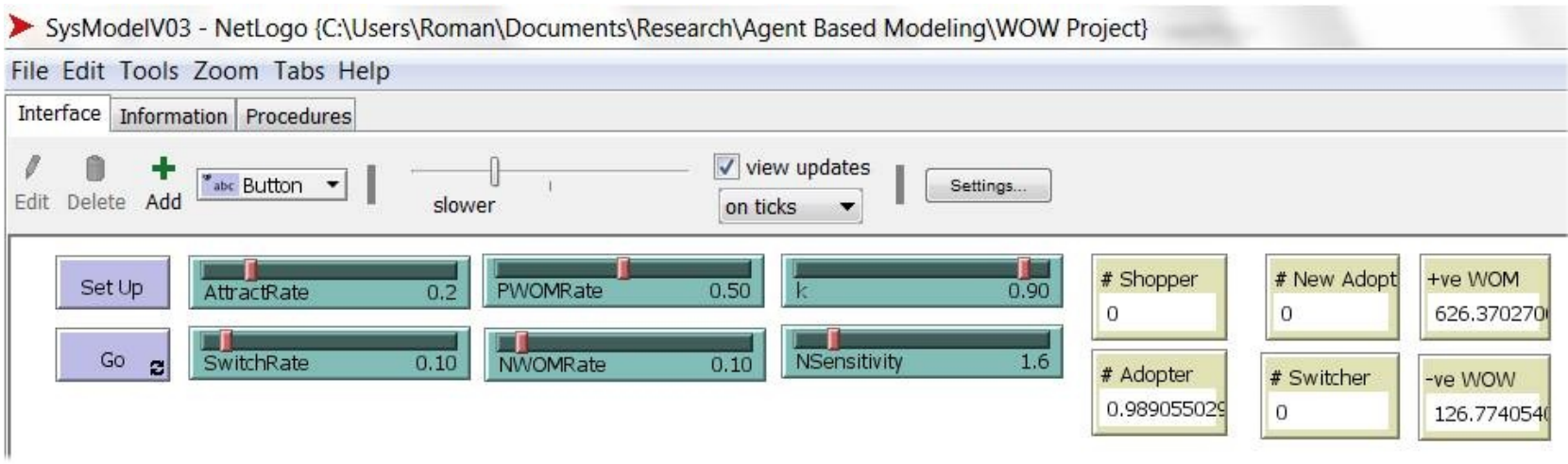

Figure 7. Sample controls used on the user interface of the system dynamics model for word-of-mouth 


\section{Word-of-Mouth and Its Effect in an Online Environment}

Roman Wong and Shirley Ye Sheng

determined by applying an appropriate probability value to new adopters (Adoption). For this study, the model was configured to use 0.4 and 0.05 on pwom and nwom, respectively. The two probability values were obtained from an earlier survey of subjects who had previous online buying experience. Figure 8 captures the change in the adoption and the number of potential adopters over time.

\section{Implications for Global Business Creation}

In this study, we aim to gain understanding about the dynamics in a word-of-mouth system by investigating the relationship between the buying decision and the decision to participate in word-of-mouth communication. The system dynamics approach can also be applied to many other business problems that are characterized by interdependence, mutual interaction, information feedback, and circular causality. One such good example is applying the approach to explore the various issues associated to the creation of global business.

In formulating business strategy for the global business, it is most important for the global entrepreneurs to possess the ability to assess the environmental factors in the global markets. These environmental factors are multi-faceted, including governmental, political, economic, social, cultural, and technological factors. The "Four Ps" of marketing (i.e., product, price, place, and promotion) for the global business are all going to be different and will become uncertain. In particular, an entrepreneur planning to create a business at the global level will face the difficulties in the following aspects:

1. Ascertaining the differences in consumer needs and their usage patterns for products

2. Managing product-mix issues because consumers in global markets may not respond at the same to marketing-mix elements as in the home market

3. Evaluating the processes for the brand and product development in a competitive environment that is unfamiliar

4. Evaluating how the differences in the legal environment may impact on the overall marketing strategy

5. Assessing how the differences in administrative procedures may impact on the overall efficiency and costing of distribution

All of the above issues are difficult to address since each of them typically involves many factors that are interdependent, relation to large amounts of information and feedback, and feature circular causal effects among the factors and outcomes. The great explanatory power and insightfulness of a system-dynamics model will help global entrepreneurs visualize and understand how results are associated with their policy actions in interactions with the various factors.

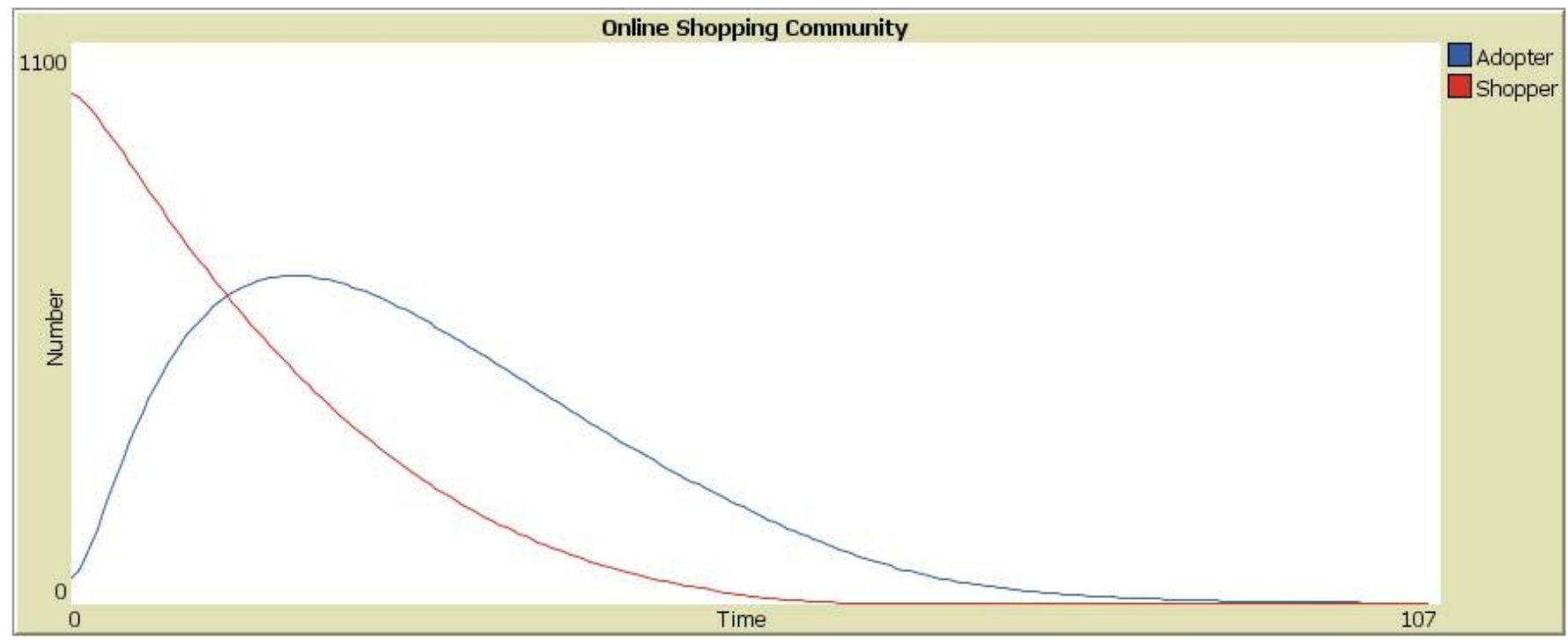

Figure 8. Number of adopters and potential adopters over time in a simulated online shopping community 


\section{Word-of-Mouth and Its Effect in an Online Environment}

Roman Wong and Shirley Ye Sheng

\section{Conclusion}

In this paper, we used a system-dynamics approach to model the recursive relationship between online shoppers' decisions to participate in word-of-mouth activities and how these activities in turn exert influence the buying decisions of other shoppers. We presented a system-level model that simulates the dynamics of such recursive relationships between the word-of-mouth decision processes. The contribution of the model we present is the tool we provide for the study of the complex dynamics in the product-adoption process and the word-of-mouth processes thereafter.

The future direction for this research is multifold. First, we shall continue our study by going into the empirical stage of systematically observing and collecting parameter data from more real-world shopping websites. Such empirical data will provide us with useful insights and help us validate our model, which can then be applied to other marketing-planning activities. The role of word-of-mouth through electronic channels in the adoption decision process is important; the understanding we will gain about word-of-mouth adoption will be valuable to marketers.

Second, we are constructing an agent-based model with adaptive agents that mimic various properties, including the behavioural ones, of online shoppers who buy and participate in online word-of-mouth activities. The objective is to observe the interaction of the agents in the community at a microscopic level and compare the emerging outcomes with the aggregate system model we constructed in this study. We believe such a comparative study to be valuable in terms of validating the models and reconciling unanswered questions arising in the simulation studies.

The system-dynamics modeling approach demonstrates a high explanatory power that helps users to explore and understand the nonlinear and dynamic nature of complex systems. In this paper, we demonstrate its use in our study of the recursive relationship of the online review activities and consumers' buying decision. It can also support the evaluation of many difficult business problems that involve the assessment of management policy in interaction with numerous environmental factors. Such complex problems include the creation of a global business.

\section{About the Authors}

Roman Wong is a professor in the areas of information systems and operations management at the Andreas School of Business of Barry University. He received his $\mathrm{PhD}$ in information systems from Southern Illinois University at Carbondale, and he received an MBA from the University of North Carolina at Charlotte. Before joining Barry University, he held a faculty position at the California State University at Northridge. His current research interests include the interrelations between the online review and product adoption processes and the development of innovativeness in emerging countries.

Shirley Ye Sheng is an Assistant Professor of Marketing at Andreas School of Business of Barry University. She received her $\mathrm{PhD}$ in Business Administration with a Marketing concentration from Florida Atlantic University in the United States, and she received a Master of Science degree in Finance from Leicester University in the United Kingdom. Her research focuses on international marketing, consumer behavior, and marketing history.

Citation: Wong, R, and S. Y. Sheng. 2012. A Business Application of the System Dynamics Approach: Wordof-Mouth and Its Effect in an Online Environment.

Technology Innovation Management Review. June 2012: 42-48. 